OPEN ACCESS

Edited by:

Sarah R. Amend,

Johns Hopkins Medicine,

United States

Reviewed by:

Robert Beckman,

Georgetown University, United States

David Basanta,

Moffitt Cancer Center, United States

${ }^{*}$ Correspondence:

Patrice Lassus

patrice.lassus@inserm.fr

Robert J. Noble

robert.noble@city.ac.uk

${ }^{\dagger}$ Present address:

Robert J. Noble,

Department of Mathematics, City

University of London, London,

United Kingdom

Patrice Lassus,

IRCM, INSERM, Université

de Montpellier, ICM, CNRS,

Montpellier, France

FThese authors have contributed equally to this work

Specialty section:

This article was submitted to Behavioral and Evolutionary Ecology,

a section of the journa

Frontiers in Ecology and Evolution

Received: 05 March 2021

Accepted: 28 June 2021

Published: 23 July 2021

Citation:

Noble RJ, Walther $V$, Roumestand C, Hochberg ME

Hibner U and Lassus P (2021)

Paracrine Behaviors Arbitrate Parasite-Like Interactions Between

Tumor Subclones.

Front. Ecol. Evol. 9:675638. doi: 10.3389/fevo.2021.675638

\section{Paracrine Behaviors Arbitrate Parasite-Like Interactions Between Tumor Subclones}

\author{
Robert J. Noble ${ }^{1,2 *}$, Viola Walther ${ }^{3}$, Christian Roumestand ${ }^{4}$, Michael E. Hochberg ${ }^{5,6}$, \\ Urszula Hibner ${ }^{3 \neq}$ and Patrice Lassus ${ }^{3 * t \neq}$
}

\begin{abstract}
${ }^{1}$ Department of Biosystems Science and Engineering, ETH Zürich, Basel, Switzerland, ${ }^{2}$ Department of Evolutionary Biology and Environmental Studies, University of Zurich, Zurich, Switzerland, ${ }^{3}$ Institut de Génétique Moléculaire de Montpellier, Université de Montpellier, CNRS, Montpellier, France, ${ }^{4}$ Centre de Biochimie Structurale INSERM U1054, CNRS UMR 5048, Université de Montpellier, Montpellier, France, ${ }^{5}$ Institute of Evolutionary Sciences, University of Montpellier, Montpellier, France, ${ }^{6}$ Santa Fe Institute, Santa Fe, NM, United States
\end{abstract}

Explaining the emergence and maintenance of intratumor heterogeneity is an important question in cancer biology. Tumor cells can generate considerable subclonal diversity, which influences tumor growth rate, treatment resistance, and metastasis, yet we know remarkably little about how cells from different subclones interact. Here, we confronted two murine mammary cancer cell lines to determine both the nature and mechanisms of subclonal cellular interactions in vitro. Surprisingly, we found that, compared to monoculture, growth of the "winner" was enhanced by the presence of the "loser" cell line, whereas growth of the latter was reduced. Mathematical modeling and laboratory assays indicated that these interactions are mediated by the production of paracrine metabolites resulting in the winner subclone effectively "farming" the loser. Our findings add a new level of complexity to the mechanisms underlying subclonal growth dynamics.

Keywords: cancer evolution, intratumor clonal heterogeneity, evolutionary game theory, parasitism, paracrine signaling, beta-hydroxybutirate, lactate, Lotka-Volterra model

\section{INTRODUCTION}

Considering tumors as complex ecosystems has led to the notion that diverse cancer cell subclones engage in heterotypic interactions reminiscent of those that operate in organismal communities (Heppner, 1984; Axelrod et al., 2006; Merlo et al., 2006; Tabassum and Polyak, 2015). Mutually negative interactions are thought to be ubiquitous in cancer (Nowell, 1976; Greaves and Maley, 2012). As in classic ecosystems, cancer cells compete for nutrients and space, and competition between emergent subclones gives rise to complex temporal and spatial dynamics of tumor composition and growth (Tabassum and Polyak, 2015). Positive ecological interactions (mutualism and commensalism) have been observed in cancer models in mice (Calbo et al., 2011; Cleary et al., 2014) and in drosophila (Ohsawa et al., 2012). In these cases, one subclone acquires new abilities, such as the capacity to grow or metastasize, only in the presence of another subclone, resulting in the tumor as a whole progressing toward a more aggressive phenotype. In contrast, the prevalence within tumors of asymmetric interactions such as amensalism, parasitism and facilitation remains an open question. Defining the mechanisms of tumor ecology is essential for a better understanding 
of cancer progression and may lead to novel therapeutic strategies (Gatenby and Brown, 2017; Maley et al., 2017).

To gain insight into molecular and cellular events related to ecological interactions between cancer subclones, we took advantage of a model described over three decades ago, based on two closely related murine cancer cell lines derived from a single spontaneous mouse mammary tumor (Dexter et al., 1978; Miller et al., 1988). When cultured separately, the two cell lines have similar growth rates, yet in co-culture one cell line (the "winner") expands at the expense of the other (the "loser"). Our careful re-examination of this model, combining experiments with mathematical modeling and parameter inference, indicated that the cellular behaviors of the two subclones are surprisingly sophisticated. Both cell lines produce paracrine metabolites that boost proliferation of the winner and also decrease the growth rate of the loser. Our results thus unveil a type of facultative parasitic behavior of the winner subclone. We further identified beta-hydroxybutyrate and lactate as metabolites that contribute to these phenotypes and characterized their modes of action. We discuss our results in the context of how previously underappreciated ecological interactions may contribute to the complexity of tumor growth dynamics.

\section{RESULTS}

\section{T07 Cells Have a "Winner" Phenotype}

Two cell lines derived from a single mouse mammary carcinoma - 168 and 4T07 cells - have similar growth rates when cultured individually, yet the 4T07 clone displays a dominant phenotype when grown together, either in cell culture or in orthotopic allografts in vivo (Miller et al., 1988). Several hypotheses to account for this interesting behavior had been tested in the original work, but the precise mechanism behind these competitive interactions has so far not been identified.

We began by verifying that in our hands the lines maintain their competitive characteristics. To facilitate lineage tracing we first generated lines stably expressing GFP, the expression of which did not alter cell growth (Figure 1A). Next, we followed growth characteristics of 4 T07 and 168FARN cells, the latter being a drug-resistant derivative of the original 168 clone (Aslakson et al., 1991), in a continuous culture for 3 weeks. The cells were seeded as 1:1 mix at a density that allowed them to reach confluence within 3-4 days, at which point they were harvested and re-seeded in a new well at the original density. Remaining cells were analyzed by flow cytometry to determine the proportion of GFP expressing clones in the expanding population.

The homotypic co-culture (same line with and without GFP) confirmed that GFP has no impact on cellular proliferation (Figures 1B, 2B). In contrast, heterotypic co-culture conditions (two different lines, one expressing GFP) revealed the dominance of the $4 \mathrm{~T} 07$ clone (Figures 1B, 2B).

These results confirm the originally described ecological interaction between the clones: 4T07 gradually dominates the culture while the 168FARN cells become scarce within 1517 days. Importantly, the dominant phenotype is independent of the starting ratio between the two cell lines (Supplementary Figures 1A,B).

\section{Co-culture Alters the Proliferation Rates of Both "Winner" and "Loser" Cells}

As originally discussed for the two clones under study (Miller et al., 1988), the expansion of a single clone in co-culture could be due to alterations in cell death or changes in the proliferation rates of either or both clones. We measured apoptosis in the loser 168FARN clone and found identical, very low levels of cell death under homotypic and heterotypic conditions (Supplementary Figure 2A). Next, we used time-lapse microscopy to assess the growth dynamics of both clones in continuous culture. The cells were seeded at a density that allowed reaching confluence in 4 days and were photographed every $45 \mathrm{~min}$ for the last 3 days. We measured the overall pixel intensity for each frame (Figure 3A) as a proxy for the growth rate of the fluorescently tagged cell line. This analysis revealed that under co-culture conditions, the growth rate of 168FARN decreased, whereas that of $4 \mathrm{~T} 07$ increased relative to mono-cultures. To test whether increased net growth of the winner population is due to the alteration of proliferation, we estimated the proportion of cells in the $S$ phase of the cell cycle by performing pulse-chase EdU staining. The results presented in Supplementary Figure 2B confirmed that heterotypic co-culture gave rise to significant decrease in cells actively replicating DNA for the loser clone and a significant increase in the winner clone. Overall, these results suggest that the dominant phenotype displayed by the winner cells in co-culture can be explained by changes in proliferation that operate in opposing directions on the winner and the loser cells.

\section{Mathematical Modeling and Inference of Evolutionary Parameter Values}

To gain further insight into the ecological interactions between the winner and loser cell types we turned to mathematical modeling. Examination of the growth curves revealed two distinct phases of evolutionary dynamics (Figures $3 \mathbf{A}, \mathbf{B}$ ). In phase 1 , from 0 to $45 \mathrm{~h}$, the two cell types grew exponentially in both homotypic and heterotypic cultures, and the growth rate of 168 was higher than that of $4 \mathrm{~T} 07$. This first phase can be regarded as a transition period before the cells start altering and responding to their new environment. By contrast in phase 2, from 45 to $72 \mathrm{~h}$, the growth curves were strongly affected by interactions within and between the two cell types, and 4T07 grew faster than 168. To enable us to determine the mode of the ecological dynamics in each phase, we opted for a parsimonious, piecewise mathematical model. Specifically, we assumed a model with exponential growth in phase 1 and a transition to densitydependent competitive Lotka-Volterra-type dynamics in phase 2.

By fitting our model to the homotypic growth curves, we inferred the values of the phase 1 and phase 2 growth rates and the within-type interaction parameters (see section "Materials and Methods"). To infer the between-type interactions, we used additional data from 72-h competition assays, covering a wide range of initial ratios of the two cell types. Although this latter 




B
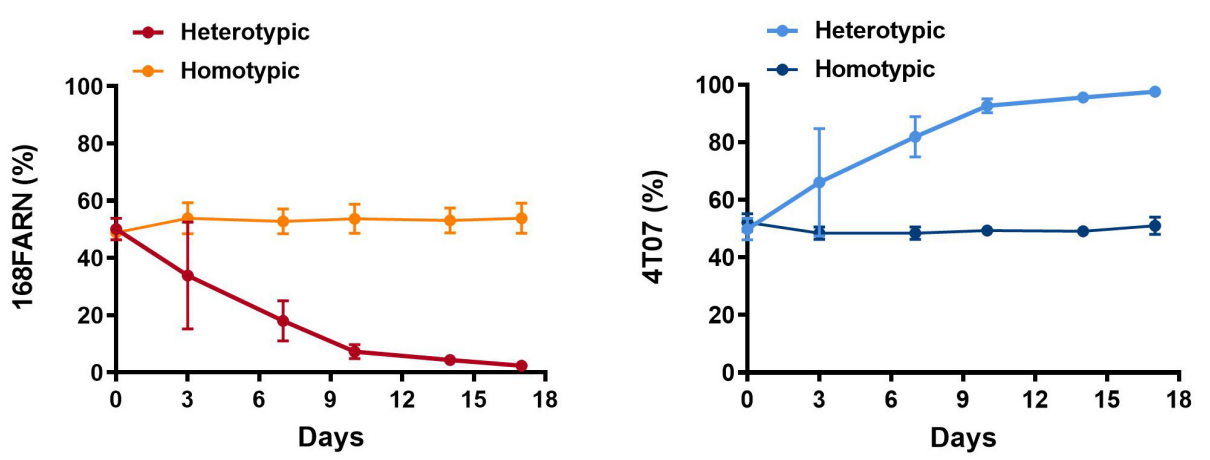

FIGURE 1 | Mutual impacts on subclonal growth. (A) 168FARN and 4T07 parental cells were transduced either with an empty retroviral vector (168P and 4T07P) or with labeled with a GFP-encoding retrovirus (168G and 4T07G). Cells were seeded in triplicate in six-well plates at a density of 50,000 cells/well and cultured for the indicated times before harvesting and counting. (B) $10^{5}$ Cells were seeded at a 1:1 ratio in homotypic (parental and GFP expressing derivative of the same cell line) or heterotypic (different cell lines, one expressing GFP) co-cultures and harvested and replated at the initial densities (10 5 cells/plate) at indicated times. The ratios of GFP-labeled to unlabeled cells were estimated by flow cytometry. The results represent data from three independent experiments and are shown as mean \pm SEM.

data set comprises only the initial and final proportions (at the beginning of phase 1 and the end of phase 2), we were able to infer the proportions at the beginning of phase 2 by adjusting for the exponential growth of both types during phase 1 . We then used these inferred proportions and our previously inferred parameter values to estimate the remaining interaction parameters (see section "Materials and Methods"). The resulting model gives a good fit to the competition assay data (Figure 2A, first column) and is consistent with heterotypic time-lapse data not used for parameter inference (Figure 3 and Supplementary Figure 6).

The inferred parameter values (Table 1) imply that during phase 2, 4T07 has a large negative effect on both itself and on 168 , consistent with $4 \mathrm{~T} 07$ producing a harmful diffusible factor. The negative effect of 168 on itself is only about half as large, and 168 has approximately zero net effect on the growth of 4T07. This suggests that ubiquitous negative effects of 168 on 4 T07 (e.g., likely due to waste products and competition for resources) are offset by positive effects, such as due to a beneficial diffusible factor. Also, during phase 2, the intrinsic growth rate of 168 (that is, the inferred growth rate before accounting for cell-cell interactions) is approximately 30\% lower than that of 4T07, consistent with the conventional hypothesis that producing beneficial factors is costly. This disadvantage is offset by 168 having an approximately 30\% higher carrying capacity (defined as the upper limit of the homotypic population size). Over phase 2 , or any longer period that includes phase 2 , the inferred net growth rate of $4 \mathrm{~T} 07$ (that is, the growth rate after accounting for cell-cell interactions) is invariably higher than that of 168 , which means 4T07 will come to dominate numerically, no matter their initial frequency.

Since we also conducted 96-h competition assays, we were able to infer the population dynamics during a third phase (72$96 \mathrm{~h}$ ). For every initial ratio of the two cell types, the growth rate difference (also known as the gain function) was on average lower in the 96-h than in 72-h competition assays (Supplementary Figure 5). Moreover, this difference did not depend on the initial ratio, which implies it was not caused by a change in interaction parameters. A parsimonious way to account for this effect is to assume a reduction in 4T07's intrinsic growth rate during phase 3, as would be expected to result from starvation and/or the build-up of toxic waste products. Making this adjustment to our model indeed produces a better fit to the competition assay data (Figure 2A, middle column, Figures 2B,C). The predicted dynamics are shown in Figures 3C,D. 


\section{A}
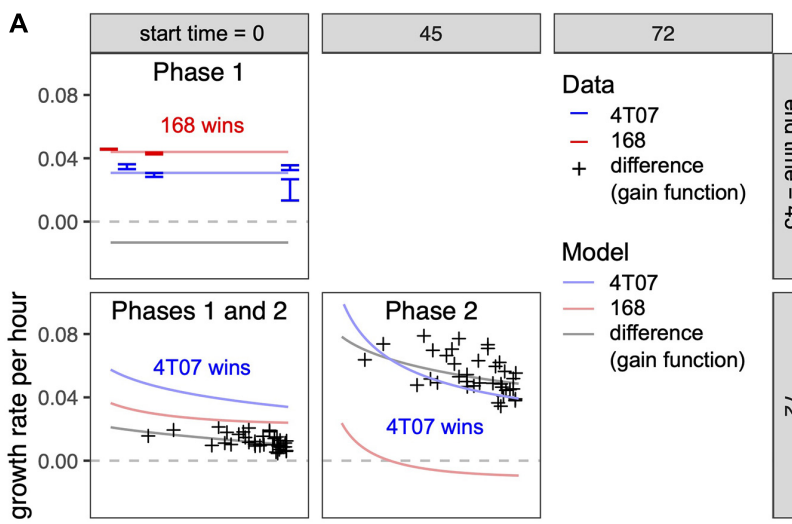

Model

- 4T07

- 168

(gain function)

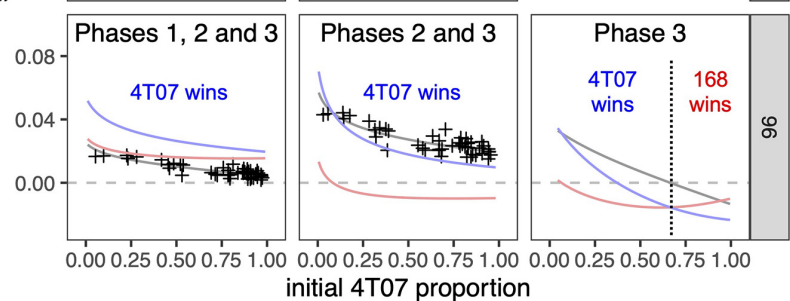

B
을
흥
을
웋
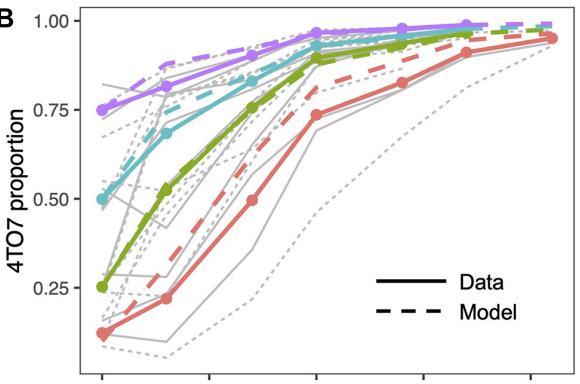

$\approx$

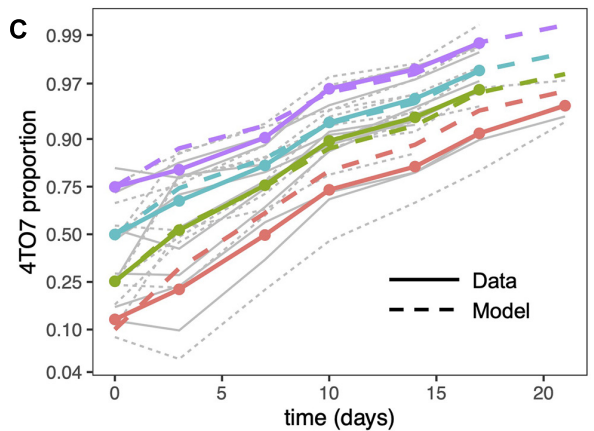

Type

- 4T07P 4T07G

Ratio

$-10-90$

$-25-75$

- 50-50

$=75-25$

Type

- 4T07P 4T07G

Ratio

- 10-90

$=25-75$

- 50-50

$=75-25$

FIGURE 2 | Mean net growth rate differences according to mathematical model and experimental data. (A) Inferred mean net growth rates and mean net growth rate differences (gain functions) over different time periods, corresponding to different phases within competition assays. Columns correspond to different start times and rows to different end times of the phase(s) under consideration. For example, the center panel labeled "Phase 2 " corresponds to the period between 45 and $72 \mathrm{~h}$. The initial 4T07 proportion (horizontal axis) is measured at the start of the respective period and the growth rate (vertical axis) is averaged over the period. Phase 1 data are from time-lapse microscopy. Other data points in the first column are from serial competition assays, such that each point corresponds to the slope of a thin gray line in panel (B). Data points in the middle column are obtained from the competition assay data by adjusting for exponential growth during phase 1 (see section "Materials and Methods"). Curves are the results of our mathematical model (see section "Materials and Methods") with parameter values inferred from data (Table 1). (B) 4T07 frequency dynamics across serial competition assays. Thick solid lines are averaged data (means of replicates with similar initial 4 TO7 proportions) and thick dashed lines are results of our mathematical model with parameter values inferred from data. Thin gray lines are data for individual experiments. A total of $10^{5}$ cells were seeded in co-cultures and harvested and replated as indicated. 4T07 parental cells were transduced either with an empty retroviral vector (4T07P) or labeled with a GFP-encoding retrovirus (4T07G). The ratios of GFP to unlabeled cells were estimated by flow cytometry.

(C) Logit-transformed 4T07 frequency dynamics. This panel shows the same data as panel (B) but with a logit-transformed vertical axis so that the slope of each curve is equal to the mean net growth rate difference (the gain function, as described in section "Materials and Methods" and Supplementary Figure 7).

Finally, having inferred all the evolutionary parameter values, we calculated net growth rates of the two cell types, averaged over different time periods. Over any period that includes phase 2 , our model predicts that the net growth rate of both cell types will decrease non-linearly with increasing initial 4T07 frequency (pink and blue curves in Figure 2A). However, the net growth rate of $4 \mathrm{~T} 07$ decreases faster than that of 168 , which is why the gain function (gray curve in Figure 2A) also decreases. In phase 3 , if the initial proportion of $4 \mathrm{~T} 07$ is high (above 70\%), then 168 has a higher net growth rate than $4 \mathrm{~T} 07$, but in this case both of the inferred net growth rates are negative. Overall, the interactions are effectively equivalent to those of a parasite and its host, such that the "loser" 168 suffers from the presence of the "winner" 4T07, while also enhancing the winner's fitness.

\section{$\beta$-Hydroxybutyrate Secreted by the Loser Clone Stimulates Winner Clone Proliferation}

To identify the molecular mechanisms at the basis of the altered growth of winners and losers when in co-culture, we first focused on the increase in proliferation rate of $4 \mathrm{~T} 07$ cells. Heterotypic culture experiments performed at low cell density suggested that the dominant effect did not require extensive cell-cell contacts (Supplementary Figure 3). We reasoned that a soluble factor secreted by 168FARN could induce a proliferation boost in 4 T07. To test this hypothesis, we collected conditioned media from each line cultured for 3 days and used each medium separately to grow $4 \mathrm{~T} 07$ for an additional $24 \mathrm{~h}$. As controls, we either left the $4 \mathrm{~T} 07$ medium after the 3 days of conditioning or replaced it with fresh medium. The results shown in Figure 2A confirm our hypothesis: the medium conditioned by 168FARN induced a significant increase in $4 \mathrm{~T} 07$ proliferation. Importantly, this effect was not due to differences of medium exhaustion by the two cell lines, since the addition of fresh medium did not boost 4T07 proliferation.

Since our data strongly suggested that a soluble factor originating from 168FARN accounted for the increase in 4T07 proliferation, we next sought to define its molecular nature. First, we separated the 168FARN-conditioned medium into high and low MW fractions with a $3 \mathrm{KDa}$ molecular cutoff column. The low MW fraction contains mainly metabolites while the high one is enriched in proteins. After complementing each fraction, respectively, with $10 \%$ serum or with DMEM to obtain full 

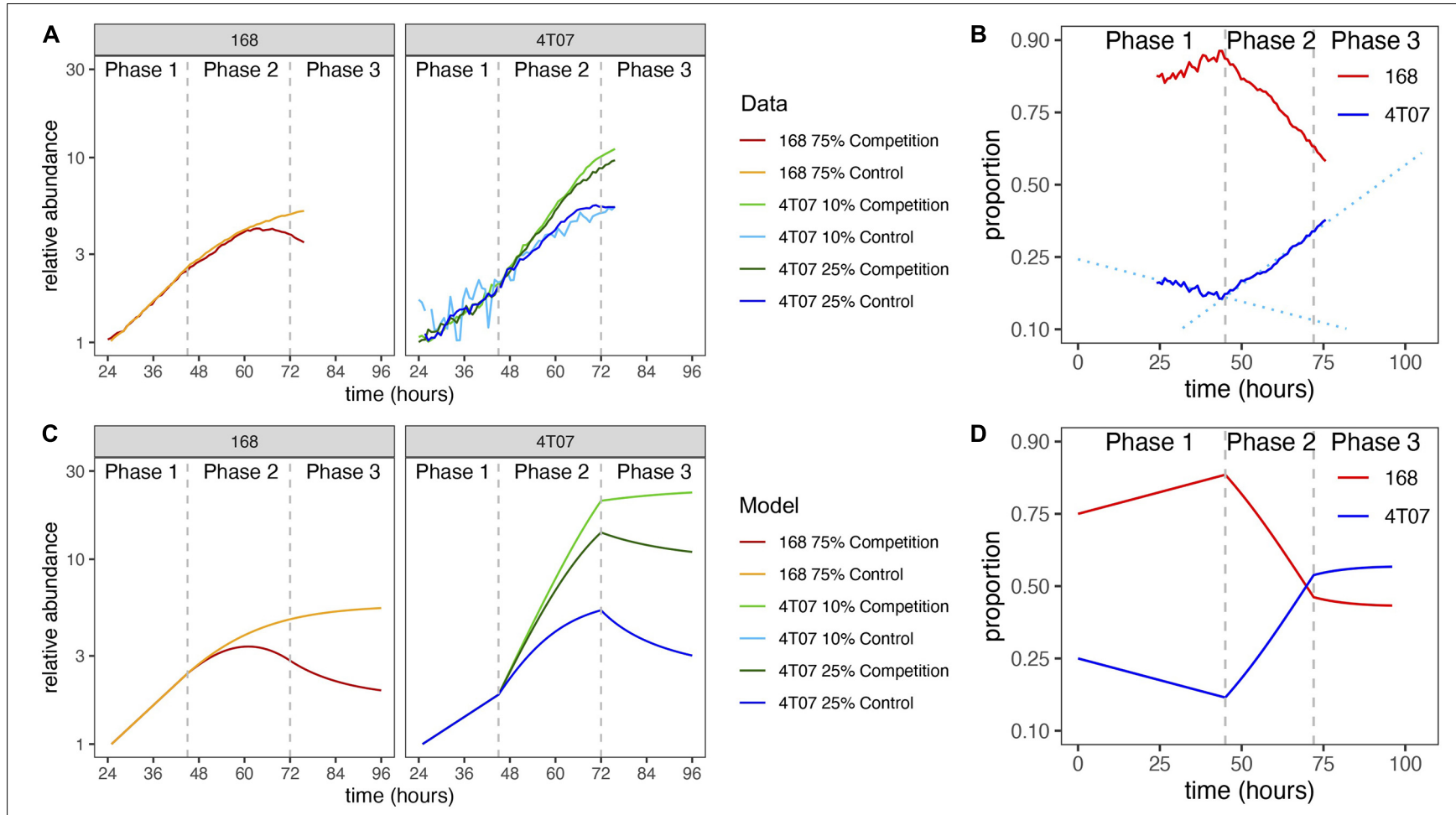

FIGURE 3 | Normalized growth curves of homotypic and heterotypic mixes of subclones. (A) The GFP fluorescence of the labeled subclone was measured by time-lapse microscopy. Cultures were seeded with $10^{5}$ cells per well. Log-transformed data were normalized by fitting regression lines and dividing by the inferred value at $24 \mathrm{~h}$. Vertical dashed lines mark the start of phase 2 (45 h) and phase 3 (72 h). (B) Frequency dynamics. Curves obtained by combining the results of two competition experiments: one with labeled 4T07 and the other with labeled 168. The initial 4T07 proportion was $25 \%$ in both cases. The vertical axis is logit-transformed so that the slope of each curve is equal to the difference in net growth rates at the corresponding time (see section "Materials and Methods"). Dotted regression lines are shown to draw attention to the change of slope. (C) Normalized growth curves according to mathematical model with parameter values inferred from data. The model is described in section "Materials and Methods" and parameter values are given in Table 1. (D) Frequency dynamics according to mathematical model with parameter values inferred from data.

media conditioned with either low or high MW secretomes, we used them in a proliferation assay as in Figure $4 \mathbf{A}$. The results (Figure $4 B$ ) of this series of experiments unambiguously identified the low MW fraction of the 168FARN-conditioned medium as the source of the pro-proliferative factor. To further explore its identity, we employed nuclear magnetic resonance spectroscopy to compare the composition of low MW fractions prepared from fresh medium and from the 168FARN- and 4T07-conditioned ones (Henke et al., 1996). Two major peaks specific for the conditioned media corresponded to a very strong signal for lactate secreted by $4 \mathrm{~T} 07$ cells, and a significant increase in a peak identified as $\beta$-hydroxybutyrate (BHB) in the 168FARNconditioned medium (Figure 5A). BHB is a ketone body mainly produced by the liver after long fasting periods and which is used by different tissues as a source of carbon to supplement the lack of glucose (Newman and Verdin, 2017). In addition, $\mathrm{BHB}$ is also produced by other cell types, such as adipocytes or cancer cells (Grabacka et al., 2016; Huang et al., 2017; Wang et al., 2017). To confirm the NMR-based identification of the $\mathrm{BHB}$ peak, we employed an enzymatic assay to measure $\mathrm{BHB}$ concentration in conditioned media from 4 T07 and 168FARN (Figure 5B). The results were in perfect agreement with the NMR analysis: BHB production is significantly higher in the loser than in the winner cell clone. To test whether this metabolite was indeed responsible for the increased proliferation of 4T07, we next complemented the medium of exponentially growing 4T07 cells with purified BHB. As shown in Figure 5C, BHB increased the $4 \mathrm{~T} 07$ proliferation rate to a level comparable to that obtained with the 168-conditioned medium. We thus conclude that loser cells increase the winner's growth rate through the secretion of BHB.

\section{Presence of the Winner Clone Stimulates $\beta$-Hydroxybutyrate Production by Loser Cells}

After assessing BHB production in homotypic cell culture, we evaluated its secretion under heterotypic conditions. We grew 168FARN alone or together with 4 T07 at a 1:1 ratio, maintaining the overall cell density constant. Surprisingly, despite the fact that under heterotypic conditions there are at least 50\% fewer loser cells (which are the main producers of $\mathrm{BHB}$, cf. Figure $5 \mathbf{B}$ ), the overall level of secreted $\mathrm{BHB}$ was higher than in the homotypic culture (Figure 5D). This suggests that either the presence of $4 \mathrm{~T} 07$ increased the production of the metabolite by 168FARN or, alternatively, that it was $4 \mathrm{~T} 07$ that produced more metabolite 
TABLE 1 | Mathematical model parameter values inferred from data.

\begin{tabular}{|c|c|c|c|}
\hline Parameter & Phase(s) & $\begin{array}{l}\text { Inferred } \\
\text { value }\end{array}$ & \\
\hline$r_{L, 1}$ & 1 & 0.044 & 168 growth rate in phase 1 (per hour) \\
\hline$r_{W, 1}$ & 1 & 0.031 & 4T07 growth rate in phase 1 (per hour) \\
\hline$r_{L, 2}$ & 2 and 3 & 0.073 & $\begin{array}{l}168 \text { intrinsic growth rate in phase } 2 \text { (per } \\
\text { hour) }\end{array}$ \\
\hline$r_{W, 2}$ & 2 & 0.102 & $\begin{array}{l}4 \text { TO7 intrinsic growth rate in phase } 2 \text { (per } \\
\text { hour) }\end{array}$ \\
\hline$r_{W, 3}$ & 3 & 0.04 & $\begin{array}{l}4 \text { TO7 intrinsic growth rate in phase } 3 \text { (per } \\
\text { hour) }\end{array}$ \\
\hline a & 2 and 3 & -0.004 & Density-dependent effect of 168 on 168 \\
\hline$b$ & 2 and 3 & -0.010 & Density-dependent effect of 4T07 on 168 \\
\hline$C$ & 2 and 3 & 0.000 & Density-dependent effect of 168 on 4T07 \\
\hline$d$ & 2 and 3 & -0.008 & Density-dependent effect of 4T07 on 4T07 \\
\hline$K_{L}=-r_{L, 2} / a$ & 2 and 3 & 17 & $\begin{array}{l}168 \text { carrying capacity, relative to initial } \\
\text { population size }\end{array}$ \\
\hline$K_{W}=-r_{W, 2} / d$ & 2 & 13 & $\begin{array}{l}\text { 4T07 carrying capacity, relative to initial } \\
\text { population size }\end{array}$ \\
\hline$\beta=b / a$ & 2 and 3 & 2.4 & $\begin{array}{l}\text { Effect of } 4 \text { TO } 7 \text { on } 168 \text {, relative to effect of } \\
168 \text { on } 168\end{array}$ \\
\hline$\gamma=c / d$ & 2 and 3 & 0.0 & $\begin{array}{l}\text { Effect of } 168 \text { on } 4 \mathrm{TO} \text {, relative to effect of } \\
4 \mathrm{TO} 07 \text { on } 4 \mathrm{TO} 0\end{array}$ \\
\hline
\end{tabular}

The interaction terms $a, b, c$, and $d$ are relative to population size, which is, in turn, relative to initial population size.

when grown in the presence of 168FARN. To distinguish between these hypotheses, we cultured both lines individually for 3 days, measured $\mathrm{BHB}$ concentration, and then exchanged the culture medium and quantified metabolite synthesis $24 \mathrm{~h}$ later. The quantification of $\mathrm{BHB}$ produced over the last day (Day 4 $\mathrm{BHB}$ concentration minus Day $3 \mathrm{BHB}$ concentration) shows that the 168FARN-conditioned medium had no effect on $\mathrm{BHB}$ secretion by $4 \mathrm{~T} 07$ cells. In striking contrast, the production of the metabolite by 168FARN more than doubled under the influence of the 4T07-conditioned medium (Figure 5E). Thus, the winner cells stimulate the losers to produce a metabolite that boosts the former's proliferation.

\section{Mechanism of $\beta$-Hydroxybutyrate Action}

We next asked about the mode of action of $\mathrm{BHB}$ on the 4T07 cells. BHB can be imported by four monocarboxylate transporters of the SLC16A gene family, the expression of which varies in different cell types. We assessed the expression of each transporter by RT-QPCR and found that MCT2, MCT3, and MCT4 were barely expressed while MCT1 was highly expressed (Figure 6A) in 4T07 cells. This result suggests that MCT1 is likely responsible for the import of $\mathrm{BHB}$ in this cell line. Interestingly, we found that MCT1 is three times more expressed in 4T07 than in 168 cells (which, like 4T07, do not express the other MCTs - Supplementary Figure 4A), suggesting that the winner cells are more efficient at taking up this metabolite than the losers (Supplementary Figure 4B). Finally, incubation of 4T07 with $\mathrm{BHB}$ upregulates MCT1, consistent with a positive feedback loop that could increase the transport of this ketone body into the dominant cell line (Supplementary Figure 4C).
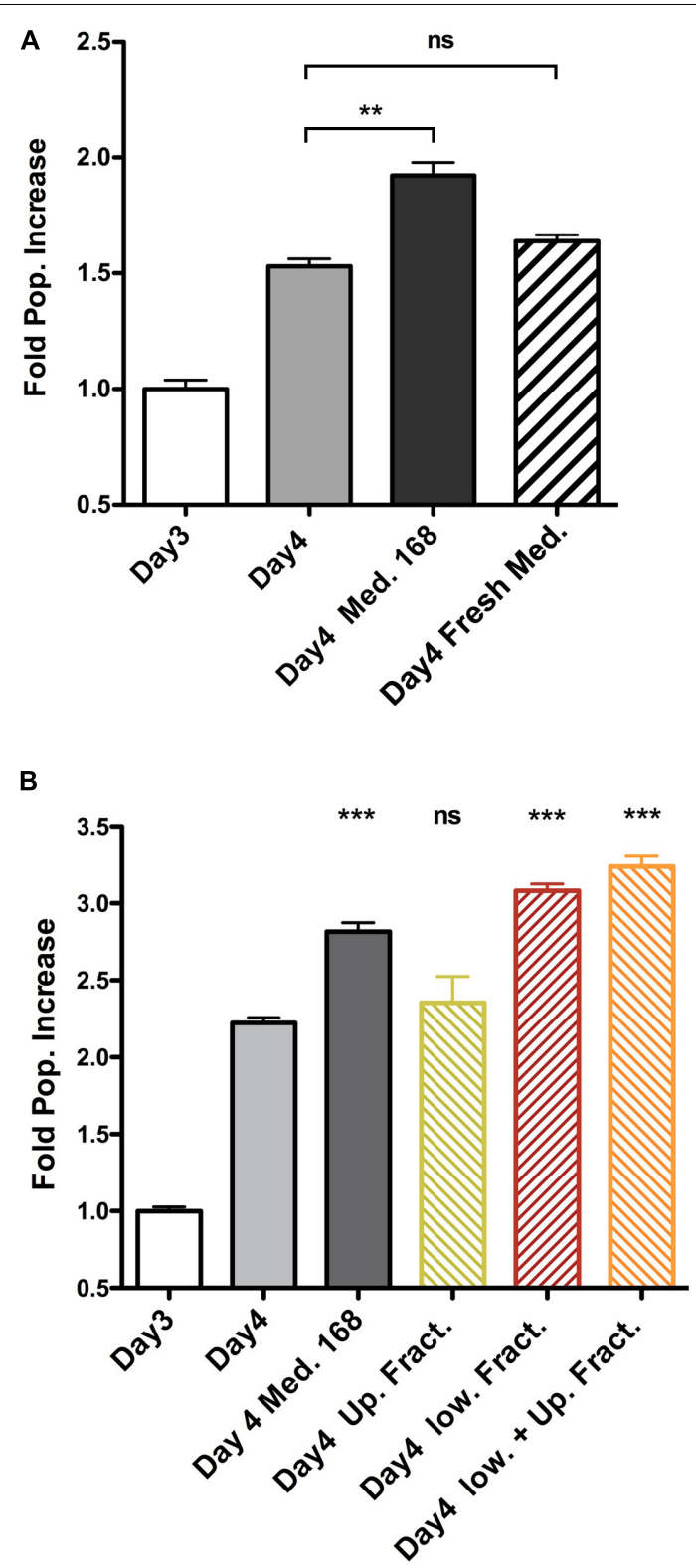

FIGURE 4 | Soluble factor secreted by 168FARN cells accelerates proliferation of the 4T07 cells. (A) 4T07 cells were grown for 3 days at which point their medium was either left unchanged, or replaced by either 168FARN-conditioned medium or fresh medium, as indicated. Cells were collected $24 \mathrm{~h}$ later and counted. Cell numbers at day 3 were arbitrarily set at 1 in order to include the data from three independent experiments. (B) The experiment was performed as in panel $(\mathbf{A})$. but the medium conditioned by 168FARN cells was fractionated by membrane ultrafiltration with a $3 \mathrm{KDa}$ molecular cutoff. After complementing the low and the high MW fractions, respectively, with $10 \%$ serum and DMEM, the media were used to grow the 4T07 cells, as in panel (A). The two fractions were also combined as a control. ns, not significant; ${ }^{* \star} p<0.01,{ }^{* \star *} p<0.001$, all compared to Day 4 point.

$\beta$-Hydroxybutyrate can be metabolized and used as a nutrient to replace glucose (Newman and Verdin, 2017). Experiments presented in Figure $\mathbf{2 A}$ show that fresh medium added at day 3 did not boost cell proliferation, suggesting that in this 
A

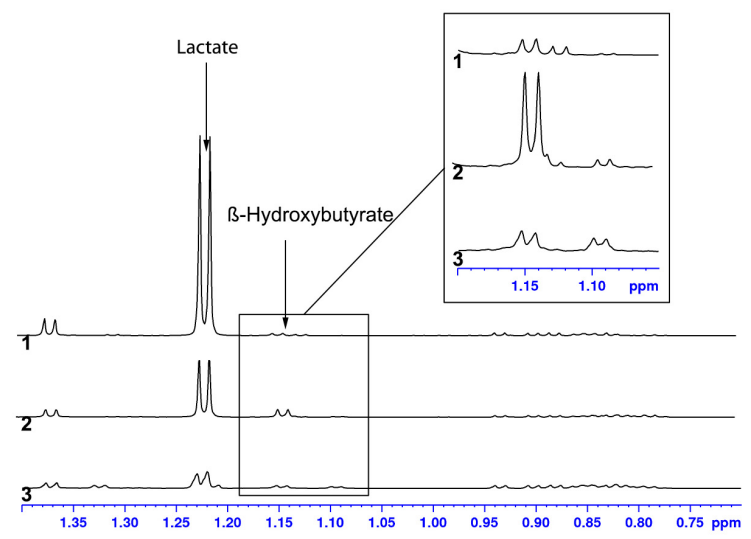

B

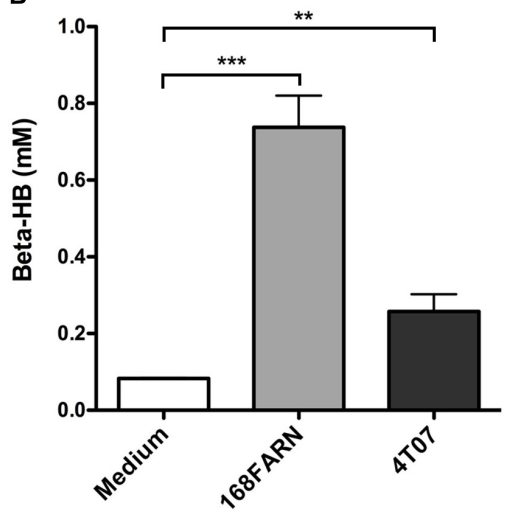

D

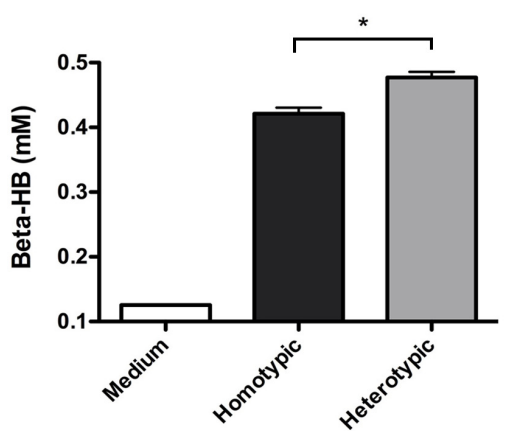

C

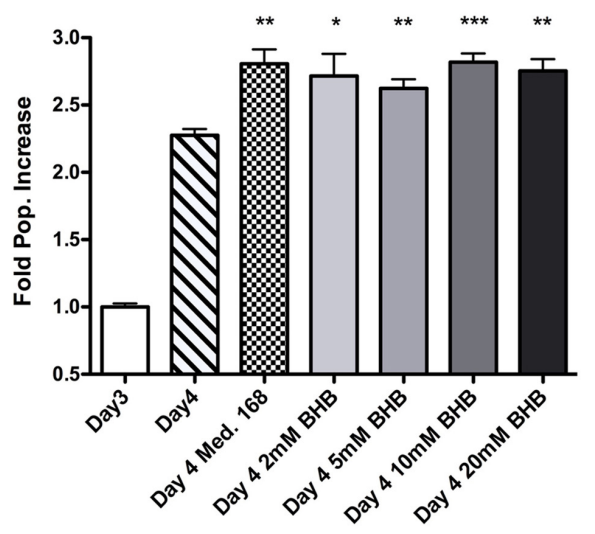

E

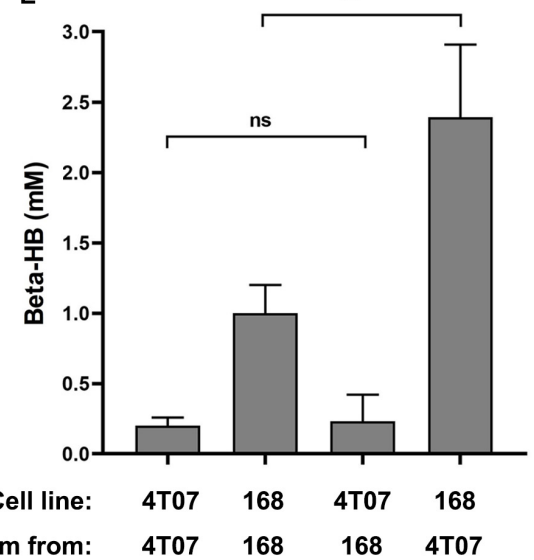

FIGURE 5 | Identification of soluble metabolites altering the heterotypic growth dynamics. (A) Superimposition of the high-field region of representative 1D proton NMR spectra recorded at $700 \mathrm{MHz}, 293 \mathrm{~K}$ and pH 7 on samples of culture media collected after growing $40 \mathrm{T7}$ cells (1) or 168FARN cells (2) for 3 days or of fresh cell culture medium (3). The arrows indicate the characteristic resonance of lactate and $\beta$-hydroxybutyrate. The insert displays a zoom in this spectral region, revealing the $\mathrm{H}$-alpha resonance of the $\beta$-hydroxybutyrate. For all spectra, peak intensities have been normalized on the intensity of the DSS resonance added as internal reference. (B) Concentration of $\beta$-hydroxybutyrate from fresh medium and from conditioned medium from 168FARN or 4T07 was quantified.

(C) Commercially available $\beta$-hydroxybutyrate at indicated concentrations was added to 4 T07 cell culture at day 3 an the growth allowed to proceed for an additional $24 \mathrm{~h}$. All points are compared to Day 4 point. (D) 168FARN alone (homotypic) or in 1:1 co-culture with 4T01 cells were grown for 4 days and extracellular $\beta$-hydroxybutyrate was measured enzymatically as in Figure 4B. (E) 168FARN and 4T07 cells were cultured individually for 3 days. The medium was then replaced by the homotypic or heterotypic conditioned one, as indicated, and the culture allowed to continue for an additional $24 \mathrm{~h}$. The $\beta$-hydroxybutyrate concentration was quantified at day 4. ns, not significant; ${ }^{*} p<0.05,{ }^{* *} p<0.01,{ }^{* * *} p<0.001$. 
experimental setup the decrease in the carbon source is not a limiting factor for growth. It is thus unlikely that $\mathrm{BHB}$ is used as an energy resource to increase proliferation rate. BHB has previously been identified as an inhibitor of class I histone deacetylases (HDAC) that modulates the expression of genes involved in reactive oxygen species detoxification (Shimazu et al., 2013). Subsequently, another group found that adipocytes use BHB to modulate the expression of a subset of genes involved in the growth of breast cancer cells (Huang et al., 2017). We thus hypothesized that $\mathrm{BHB}$ might increase the growth rate of winners through the inhibition of HDACs, thereby modulating the expression of genes involved either in ROS detoxification or in the induction of pro-proliferative factors. In support of this idea, incubation of 4T07 cells either with 168FARNconditioned medium or with purified $\mathrm{BHB}$ increased $\mathrm{H} 3 \mathrm{~K} 9$ acetylation, albeit to a lesser extent than butyrate, a bona fide HDAC inhibitor (Figure 6B).

While we could not detect in $4 \mathrm{~T} 07$ cells any modification of expression of ROS detoxification genes reported to be regulated by $\mathrm{BHB}$ in other cellular models (Shimazu et al., 2013), both BHB and 168-conditioned medium led to significant transcriptional activation of interleukin-11 (IL-11) and lipocalin 2 (LCN2) (Figure 6C). Both genes have been previously described to promote cancer cell growth and to be regulated by $\mathrm{BHB}$ through its action on HDAC activity (Yang and Moses, 2009; Grivennikov, 2013; Huang et al., 2017). Thus, our data point to the molecular mechanisms involving direct proliferation signaling.

\section{Lactate Secretion Slows Down Loser Cell Proliferation}

In addition to the positive effect of the 168FARN cells on the proliferation rate of the $4 \mathrm{~T} 07$ clone, the data shown in Figure 2 indicate that the latter negatively influences the 168FARN growth dynamics. The NMR analysis highlighted strong lactate production (see Figure 5A). This is consistent with our observation of the media color change during culture of the two lines, indicating that the winner clone has a glycolytic type of glucose metabolism leading to a rapid medium acidification in culture. Because extracellular acidification can be detrimental for cell growth, we next asked if 168FARN were particularly sensitive to such growth conditions. We quantified medium acidification by seeding cells at different densities and measuring the extracellular $\mathrm{pH}$ after 3 days of culture (Figure 7A). As expected, we found that 4T07 cells acidify the medium faster and attain a lower $\mathrm{pH}$ during culture compared to 168FARN cells. Indeed, $\mathrm{pH}$ ranged from $6.94+/-0.005$ (lowest density) to $6.79+/-0.003$ (highest density) for the winner line and from $7.38+/-0.008$ to $6.92+/-0.006$ for 168 FARN. To test whether 4T07-mediated extracellular acidification influenced 168FARN growth, we set up a proliferation assay for 168FARN cells grown in medium conditioned by the low and the high density grown 4T07 cells. To control for the effect of $\mathrm{pH}$ in the conditioned media, we included a treatment in which the medium from 4T07 was buffered at $\mathrm{pH} 7.0$ by sodium bicarbonate. These experiments revealed that the medium from the low density 4 T07 cells


C
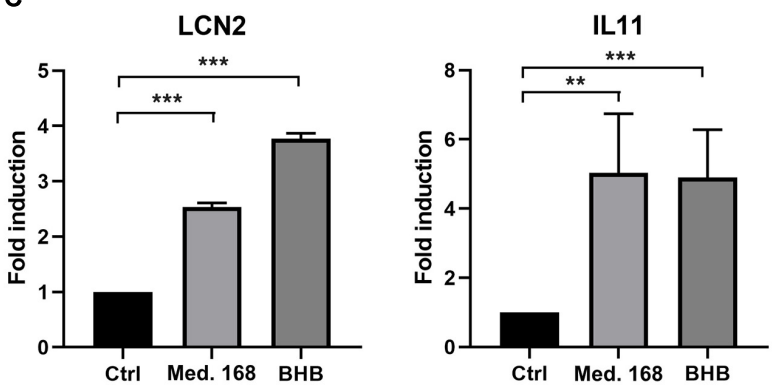

FIGURE 6 | Extracellular $\beta$-hydroxybutyrate leads to increased H3K9 histone acetylation and altered gene expression in 4T07 cells. (A) Expression levels of the slc16A family transporter genes in 4T07 were analyzed by RT-QPCR. Expression of HPRT served as normalization of the data. (B) H3K9 histone acetylation was analyzed by immunoblotting of extracts of 4T07 cells grown for $24 \mathrm{~h}$ in control, 168-conditioned medium or medium complemented with $\beta$-hydroxybutyrate or with butyrate, as indicated. Total histone $3(\mathrm{H} 3)$ abundance served as normalization control. (C) 4T07 cells cultured for 3 days were incubated for $8 \mathrm{~h}$ with 4T07- (Ctrl) or 168-conditioned medium or purified $\beta$-hydroxybutyrate $(10 \mathrm{mM})$ added to fresh medium. Total RNAs were purified and subjected to RT-QPCR with specific primers for LCN2 and IL-11. ${ }^{* *} p<0.01,{ }^{* * *} p<0.001$.

( $\mathrm{pH}$ 6.94) had no effect on 168FARN proliferation. In contrast, the medium from the high density $4 \mathrm{~T} 07$ ( $\mathrm{pH}$ 6.79) drastically decreased the 168FARN growth rate. Moreover, buffering the same medium at $\mathrm{pH} 7.0$ restored the proliferative capacity of 168FARN culture (Figure 7B). We conclude that the loser clone is indeed highly sensitive to medium acidification. Taken together our data suggest that the decrease in the growth rate of 168FARN observed in heterotypic conditions is triggered by 4T07 mediated extracellular acidification. 




B



Starting Cell number

FIGURE 7 | Impact of extracellular pH on the loser clone growth. (A) 168FARN and 4T07 cells were seeded at the indicated initial densities in six-well plates and cultured for 3 days. Culture media were removed, immediately covered with a layer of mineral oil to prevent oxidation and the $\mathrm{pH}$ was measured. (B) $10^{5}$ 168FARN cells were grown for 3 days. Medium was then replaced by conditioned media from cultures grown at low or high density, as indicated. Where indicated, $5 \mathrm{mM} \mathrm{NaCO}_{3}$ was used to buffer the 4T07 conditioned medium to $\mathrm{pH} 7$. Twenty-four hours later cells were harvested and counted. Data are from three independent experiments conducted in triplicates. ns, not significant; ${ }^{\star \star *} p<0.001$.

\section{DISCUSSION}

Heterogeneity is a ubiquitous feature of tumors that influences growth and metastasis, and thus the potential for therapeutic success. Ecological interactions between subclones are key to the emergence of this heterogeneity, yet only few empirical studies have characterized the nature of these interactions or their underlying mechanisms. These include commensal (Kaznatcheev et al., 2019; Farrokhian et al., 2020) and cooperative (Cleary et al., 2014) interactions in vitro, and how such interactions can drive tumor invasion (Chapman et al., 2014) and metastasis in vivo (Janiszewska et al., 2019; Naffar-Abu Amara et al., 2020).

Our study extends previous work (Robinson and Jordan, 1989; Marusyk et al., 2014; Archetti et al., 2015) by demonstrating that two cell lines derived from the same tumor exhibit a sophisticated relationship, whereby one (the "winner") effectively farms the population of the other (the "loser"). We further identified key metabolites (BHB and lactate) that regulate these interactions between the winning and losing clones. Similar to Archetti et al. (2015), we found that exploitative clonal interactions evolve through time, but whereas these authors observed a frequencydependent change that could explain clonal coexistence, we were unable to detect this effect. Simple mathematical analysis within the framework of evolutionary game theory nevertheless shows that, when accounting for microenvironmental heterogeneity, our inferred parameter values are plausibly consistent with longterm clonal coexistence (see section "Materials and Methods").

Because our in vitro experiments simplify the diverse, complex interrelationships that predominate in spatially complex microenvironments, the parameter values we have inferred may not precisely translate to in vivo contexts. For example, the scenario of our experimental model, which depends on microenvironmental acidification by the winner clone, may be less relevant to micrometastases that are small enough to maintain physiological pH (De Palma et al., 2017; Beckman et al., 2020). On the other hand, there is an overwhelming consensus that in larger tumors (both primary and metastatic), neoangiogenesis produces abnormal, leaky vessels that give rise to poor oxygenation and acidic conditions (De Bock et al., 2011), consistent with our experimental system. That paracrine signaling is responsible for the effects we observed between winner and loser cell lines suggests that the spatial arrangement of these cells could be crucial to their growth and relative frequencies in situ (Archetti et al., 2015). The effect of spatial structure would depend on the typical distance that secreted molecules travel through the complex tumor microenvironment. Our results indicate that areas of contact or close proximity between the two subclones will grow faster and therefore come to dominate spatially isolated populations, producing what is effectively a mixed 4T07-168FARN "phenotype." The actual spatial arrangement of these two subclones in the original tumor is unknown, but the authors of the study originally isolating these cell lines note that they may represent only a small sample of the tumor's diversity (Dexter et al., 1978). A growing body of evidence suggests that single, site-specific biopsies may be of little use in quantifying spatial heterogeneity, due to the multiscale (local, regional, metastatic) nature of tumor evolution (Amirouchene-Angelozzi et al., 2017). Computational modeling indicates that the range of cell-cell interaction and the mode of cell dispersal are crucial factors determining the pattern of intratumor heterogeneity and associated characteristics of tumor growth and evolutionary potential (Waclaw et al., 2015; Noble et al., 2020). While a comprehensive description of intra-tumoral ecological interactions is a daunting task, beyond the power of existing technology, a fuller understanding of their general features is essential for devising therapies aimed at rendering cancer a chronic, controllable disease (Gatenby and Brown, 2020; Viossat and Noble, 2021).

We find that the complex interactions between the 4T07 and 168FARN cells are governed by paracrine signaling emanating from both clones. This mechanistic conclusion differs from the 
original observations reported by Miller et al. (1988). Indeed, in the original publication the results concerning the inhibitory effect of 4T07 conditioned media on 168 cells were inconclusive. This apparent discrepancy could be due to slightly different culture conditions used in the two sets of experiments. Indeed, the medium acidification due to the lactate release by the $4 \mathrm{~T} 07$ that is responsible for slowing down the growth of 168 cells reaches the required threshold value only after prolonged culture (3-4 days under our experimental conditions). It is thus possible that in the original report the culture time and/or the cell density were insufficient for the clear visualization of the paracrine effect of the winners on the losers. Moreover, Miller et al. (1988) did not investigate the paracrine effect exerted by the 168 on the $4 \mathrm{~T} 07$ cells. Our results are the first to show the reciprocal effects of both cell lines on each other, thus highlighting the complexity of their mutual interactions.

We have identified a ketone body, BHB, which is produced by loser cells and acts to increase the growth rate of winner cells. Mechanistically, the competitive advantage afforded by BHB to the winner clone appears to be mediated through the HDAC-controlled activation of a genetic program that boosts its proliferation. Ketone bodies are small lipid-derived molecules, physiologically produced by the liver and distributed via the circulation to metabolically active tissues, such as muscle or brain (Newman and Verdin, 2017), where they serve as a glucose-sparing energy source in times of fasting or prolonged exercise. Recently, several studies reported that cell types such as adipocytes, intestinal stem cells or cancer cells originating from colorectal carcinoma or melanoma can also produce BHB (Grabacka et al., 2016; Huang et al., 2017; Shakery et al., 2018; Cheng et al., 2019). Our results identifying BHB as a signaling molecule involved in intra-tumoral clonal interactions fall into the general category of these novel roles for ketone bodies in cell communication.

However, the link between ketone bodies and tumor development remains controversial. On the one hand, it was shown that ketonic diet slows down tumor development in brain cancer mice models (Poff et al., 2013, 2014). On the other hand, our results together with other recent data (Huang et al., 2017) suggest that BHB may favor breast cancer progression. One unexplored possibility to explain these contradictory observations is that this ketone body can be used differently by different cancer cell types, for example as a carbohydrate supply or as a HDAC inhibitor, ultimately leading to cancer-type and context specific response.

In our experimental model, BHB increases winner cells proliferation by activating a genetic program through HDAC inhibition. Among the genes we discovered to be activated by the ketone body, IL-11 is an interleukin that displays a pro-proliferative activity (Grivennikov, 2013). Interestingly, in a distinct breast cancer cell cooperation model, sub-clonal expression of IL-11 favors the expansion not only of cells that express it, but also of other cellular sub-clones (Marusyk et al., 2014). This suggests that IL-11 acting in either paracrine or autocrine fashion could lead, respectively, to cooperation or to competition between subclones, thus participating actively in the selection and evolution of tumor heterogeneity.
Overall, our experimental data therefore suggest a model in which the winner line stimulates the production of and benefits from a compound delivered by the loser line and, conversely, the loser is negatively influenced by the presence of winners through secretion of another compound.

We note that while in artificially maintained conditions of non-constrained growth (in culture) the losers are eventually eliminated, many additional selective pressures that may affect clonal fitness operate in vivo. These involve cellular response to physical cues due to crowding (Vishwakarma and Piddini, 2020) and interactions with the extracellular matrix (Lu et al., 2012) as well as response to signaling from the stroma, including its inflammatory and immune components (Quail and Joyce, 2013). These elements are expected to influence the outcome of the direct interactions between the tumoral clones and may change the nature of their ecological interaction from net exploitation (in vitro) to mutual benefit (in vivo). Future study should evaluate whether parasitic effects are observed in vivo, and determine the extent to which these cell-cell interactions mediate important tumor characteristics, including growth, drug resistance, and metastatic behavior.

\section{MATERIALS AND METHODS}

\section{Cell Culture}

4T07 and 168FARN were a kind gift of Robert Hipskind. All cell lines were cultured in Dulbecco's modified Eagle medium containing $10 \%$ fetal bovine serum, $100 \mathrm{ng} / \mathrm{mL}$ streptomycin, and $100 \mathrm{U} / \mathrm{mL}$ penicillin at $37^{\circ} \mathrm{C}$ with $5 \% \mathrm{CO} 2$.

For co-culture experiments a mixture of GFP-labeled and parental cells (empty-vector transduced) cells were seeded at the final density of $10^{5}$ cells/well in six-well plates, except where mentioned otherwise. Upon reaching confluence (3-4 days) they were harvested, diluted to the original density and replated. The remaining fraction was analyzed by flow cytometry.

\section{Immunoblot Analysis}

Cells were lysed in boiling Laemmli buffer supplemented with protease inhibitors, then sonicated and complemented with DTT. Protein concentration was determined by BCA (Thermo Scientific) assay. Fifteen to twenty micrograms of total protein were loaded onto SDS-PAGE gels and transferred onto nitrocellulose membranes. The membrane was blocked with TBST ( $1 \times$ TBS with $0.1 \%$ Tween 20$)+5 \%$ milk at room temperature for $1 \mathrm{~h}$ and incubated with primary antibody and then with horseradish peroxidase (HRP)-coupled secondary antibody (Amersham, Piscataway, NJ, United States). Activity was visualized by electrochemiluminescence. Antibodies used in this study are anti-Histone H3 (Cell signaling Technology \#9717) and anti-Acetyl-Histone H3 (Lys9) (Cell signaling Technology \#9649).

\section{Reverse Transcription and Real-Time PCR}

Total mRNA was isolated using a RNeasy mini kit (Qiagen, Germantown, MD, United States). Reverse transcription 
was performed with random hexamers and M-MLV Reverse Transcriptase (Invitrogen). Real-time PCR was performed in triplicates with LC FastStart DNA Master SYBR Green I on a LightCycler rapid thermal cycler system (Roche Diagnostics, Mannheim, Germany), according to the manufacturer's instructions. Housekeeping gene HPRT was used for normalization. Primers sequences are available upon request.

\section{Time-Lapse Microscopy}

Time-lapse microscopy was performed at $37^{\circ} \mathrm{C}$ with $5 \% \mathrm{CO} 2$, with images taken at 45 -min intervals using an inverted Zeiss Axio-Observer microscope. The images were processed and analyzed using ImageJ software.

\section{EdU Staining}

Cells were incubated with $10 \mu \mathrm{M}$ EdU for $2 \mathrm{~h}$, harvested and processed using the Click-iT ${ }^{\mathrm{TM}}$ EdU Alexa Fluor ${ }^{\mathrm{TM}} 647$ Flow Cytometry Assay Kit (Thermo Fisher Scientific \#C10424) following manufacturer instructions. Labeled cells were then analyzed on a FACSCalibur flow cytometer using CellQuest Pro software (BD Biosciences).

\section{Apoptosis Quantification}

To determine the percentage of apoptotic cells with externalized phosphatidylserine (PS), adherent and floating cells were collected and labeled with the Annexin V-Cy3 Apoptosis Detection Kit (Abcam, Cambridge, United Kingdom, \#ab14143) according to the manufacturer's instructions. Labeled cells were then analyzed on a FACSCalibur flow cytometer using CellQuest Pro software (BD Biosciences).

\section{$\beta$-Hydroxybutyrate Quantification}

$\beta$-Hydroxybutyrate concentration was measured by an enzymatic kit (Sigma-Aldrich MAK041) following the manufacturer instructions. Briefly, $\beta$-hydroxybutyrate present in the culture medium was determined by a coupled enzyme reaction, resulting in a colorimetric $(450 \mathrm{~nm})$ product, proportional to the $\beta$-hydroxybutyrate concentration. The absorbance was measured on a spectrophotometer.

\section{Medium Fractionation}

In order to separate low molecular weight molecules from the conditioned culture medium, 5-10 ml were loaded on a Vivaspin Turbo 15 PES, 3,000 MWCO column (Sartorius VS15T91) and centrifuged at $4000 \times g$ for $30 \mathrm{~min}$ following the manufacturer instructions. Both fractions were then used for subsequent experiments and RMN analysis.

\section{RMN Analysis}

NMR experiments were recorded at $293 \mathrm{~K}$ and $\mathrm{pH} 7$ on an AVANCE III BRUKER spectrometer operating at $700 \mathrm{MHz}$ (proton frequency), using a Z-gradient shielded TCI 1H-13C$15 \mathrm{~N}$ cryoprobe. Fully relaxed $1 \mathrm{D} 1 \mathrm{H}$ spectra were aquired with the regular $1 \mathrm{D}$ NOESY, using $5 \mathrm{~s}$ as relaxation delay. The samples consisted on $1.5 \mathrm{~mL}$ of cell media (fresh or conditioned by cell culture), lyophilized and dissolved in $500 \mu \mathrm{L}$ of deuterated phosphate buffer $(50 \mathrm{mM}, \mathrm{pH}$ 7). DSS (EURISOTOP ${ }^{\odot}$, final concentration: $0.5 \mathrm{mM}$ ) was added as internal reference for chemical shift referencing and as a concentration standard for spectra normalization. The assignment of the $1 \mathrm{H}$ resonances of the compound of interest in this study (lactate, $\beta$-hydroxybutyrate) was based on chemical shifts reported on the literature (1) and further confirmed using $2 \mathrm{D}[1 \mathrm{H}, 1 \mathrm{H}]$ (TOCSY) and [1H-13C] (HSQC, HSQC-TOCSY) NMR spectroscopy.

\section{Statistical Analysis}

Experiments were repeated at least three times. Data are presented as mean \pm SEM. An Independent Student's $t$-test was performed to analyze the assay results; a two-tailed Student's $t$-test was used to compare the intergroup differences. Significance was accepted for values where $P \leq 0.05\left(^{*}\right), P \leq 0.01$ $(* *), P \leq 0.001\left(^{* * *}\right)$.

\section{Overview of Mathematical Methods}

Our aim is to determine the general nature of the evolutionary dynamics in a form that can be readily compared to other systems (as opposed to generating quantitative predictions for our particular system). Accordingly, we chose to fit a simple, standard model to each distinct phase of the dynamics, such that the inferred parameter values have straightforward ecological interpretations. A key advantage of our method is that it is generic; in principle, the same method can be applied to any experimental evolution set-up with two competing populations of cancer cells, bacteria, or other entities.

This mathematical approach is in the same vein as that of Kaznatcheev (2017) and Kaznatcheev et al. (2019) but with three important differences. First, our method can accommodate a smaller data set and is thus more economical because we mostly rely on measurements of initial and final proportions in competition assays, such as can be determined via flow cytometry, rather than extensive time-lapse image analysis. Second, whereas Kaznatcheev (2017) and Kaznatcheev et al. (2019) confine their analysis to exponential or logistic growth phases, we also examine phases in which cell populations exhibit non-logistic dynamics. Third, because we consider non-logistic growth phases, we use a density-dependent rather than a frequency-dependent model.

We note that to make quantitative predictions of outcomes in different scenarios, we would require a different type of model with equations describing the dynamics of paracrine factors mediating clonal interactions. This more complicated model would include several more parameters and design choices (for example, how each paracrine factor's production rate and its effects vary with its concentration) and would thus be nonidentifiable in the absence of detailed paracrine concentration measurements. Obtaining such measurements remains as a challenge for future studies.

\section{Definitions and Mathematical Relationships}

We define the intrinsic growth rate as the exponential growth rate in the absence of interactions. In the Lotka-Volterra differential 
equations, this parameter is multiplied by the population size of the respective type. The intrinsic growth rate is the limit of the net growth rate as the population sizes approach zero (when interaction terms are negligible).

We define the net growth rate as the actual rate of change of the population size (i.e., the time derivative), which is the sum of the basic growth rate and interaction terms.

Supplementary Figures 6, 7 illustrate some of the mathematical relationships relevant to our methods.

\section{Dynamical Models and Inference From Homotypic Growth Curves}

We describe the exponential phase 1 dynamics as

$$
\frac{d L}{d t}=L r_{L, 1}, \frac{d W}{d t}=W r_{W, 1}
$$

where $L$ (loser) and $W$ (winner) are the population sizes of 168 and 4T07, respectively, and $r_{L, 1}$ and $r_{W, 1}$ are the respective growth rates.

In phase 2, we assume a density-dependent competitive Lotka-Volterra model, parameterized in terms of intrinsic growth rates $r_{L, 2}$ and $r_{W, 2}$ and interaction terms $a, b, c$ and $d$ :

$$
\frac{d L}{d t}=L\left(r_{L, 2}+a L+b W\right), \frac{d W}{d t}=W\left(r_{W, 2}+c L+d W\right) .
$$

In the homotypic case, terms $b W$ and $c L$ vanish and the phase 2 model is equivalent to logistic growth. We combine the two models and fit to the normalized time-lapse data for the homotypic growth curves using least-squares with $\mathrm{R}$ package deSolve (Soetaert et al., 2010) to infer the values of $r_{L, 1}, r_{W, 1}, r_{L, 2}$, $r_{W, 2}, a$, and $d$.

In phase 3, we assume the same model as in phase 2 except we replace $r_{W, 2}$ by $r_{W, 3}$ to account for the change in the $4 \mathrm{~T} 07$ net growth rate (equivalent to adding a densitydependent death rate).

\section{Inferring Between-Type Interaction Terms}

To infer the interaction parameters $b$ and $c$ we need data that covers a wide range of proportions of the two cell types. Since our time-lapse data is limited to only a few initial conditions, we fit the model to the outcomes of serial competition assays, and we employ the heterotypic time-lapse data for validation only. First we define

$$
\begin{aligned}
& l=\frac{L}{W+L}, w=\frac{W}{W+L}, \\
& s=\log \frac{w}{l}=\log \frac{w}{1-w}=\operatorname{logit}(w) .
\end{aligned}
$$

The time derivative of the $s$ is then equal to the net growth rate difference, which in phase 2 is

$$
\frac{d s}{d t}=r_{W, 2}-r_{L, 2}+(d-b) W+(c-a) L .
$$

In the limit $w \rightarrow 1$, the final term $(c-a) L$ is negligible and we can obtain $b$ in terms of $\frac{d s}{d t}, W$, and parameters whose values we have already inferred, as follows:

$\frac{d s}{d t}=r_{W, 2}-r_{L, 2}+(d-b) W \Rightarrow b=\frac{\frac{d s}{d t}-r_{W, 2}+r_{L, 2}}{W}+d$.

To obtain $W$, we note that in the limit $w \rightarrow 1$,

$$
\frac{d W}{d t}=W\left(r_{W, 2}+d W\right)
$$

which is the logistic differential equation with solution

$$
W(t)=\frac{W\left(t_{1}\right) r e^{r t}}{r-W\left(t_{1}\right)\left(e^{r t}-1\right) d},
$$

where $r=r_{W, 2}$ and $t_{1}$ is the time at which phase 2 begins. We can thus use our previously inferred parameter values to obtain $W(t)$ at every time $t$ in phase 2 (note that if there were not an analytical solution then we could have solved the equation numerically).

Since $W$ and $\frac{d s}{d t}$ are linearly related, we can replace them by their mean values:

$$
\begin{aligned}
& \frac{\operatorname{mean}\left(\frac{d s}{d t}\right)-r_{W, 2}+r_{L, 2}}{\operatorname{mean}(W)}+d \\
& =\frac{\operatorname{mean}\left(\frac{d s}{d t}\right)-r_{W, 2}+r_{L, 2}}{\operatorname{mean}\left(\frac{\frac{d s}{d t}-r_{W, 2}+r_{L, 2}}{b-d}\right)}+d=b .
\end{aligned}
$$

Using the mean values to calculate $b$ is convenient as our competition assays reveal only the initial and final values of $s$. Specifically, we take the means in the interval $\left[t_{1}, t_{2}\right]$, where $t_{2}$ is the time at which phase 2 ends and

$$
\operatorname{mean}\left(\frac{d s}{d t}\right)=\frac{s\left(t_{2}\right)-s\left(t_{1}\right)}{t_{2}-t_{1}}=\frac{\Delta s}{\Delta t} .
$$

It remains only to obtain the value of the above expression known as the gain function - in the limit $w\left(t_{1}\right) \rightarrow 1$. From competition assay data, we can immediately obtain $s\left(t_{2}\right)=\log \frac{w\left(t_{2}\right)}{1-w\left(t_{2}\right)}$ for each value of $s(0)=\log \frac{w(0)}{1-w(0)}$. To infer $w\left(t_{1}\right)$ and $s\left(t_{1}\right)$, we need to adjust for the exponential growth of both cell types during phase 1:

$$
\begin{gathered}
s\left(t_{1}\right)=s(0)+t_{1}\left(r_{W, 2}-r_{\mathrm{L}, 2}\right) \\
\Longrightarrow \operatorname{logit}\left(w\left(t_{1}\right)\right)=\operatorname{logit}(w(0))+t_{1}\left(r_{W, 2}-r_{L, 2}\right) \\
\Longrightarrow w\left(t_{1}\right)=\operatorname{logit}^{-1}\left(\operatorname{logit}(w(0))+t_{1}\left(r_{W, 2}-r_{L, 2}\right)\right) .
\end{gathered}
$$

We thus obtain the values of $s\left(t_{1}\right)$ and $w\left(t_{1}\right)$ in each competition assay. Finally, we determine by linear regression the relationship between $\Delta s / \Delta t$ and $w\left(t_{1}\right)$ (Supplementary figure 5B) and, from the equation of the regression line, infer the value of $\Delta s / \Delta t$ in the limit $w\left(t_{1}\right) \rightarrow 1$. We then have everything required to infer the value of $b$. By an analogous method (switching $L$ and $W, b$ and $c$, and $a$ and $d$ ) we also infer the value of $c$. 


\section{Excluding Results of First-Round Competition Assays}

In our regression to determine the relationship between $\Delta s / \Delta t$ and $w\left(t_{1}\right)$, we excluded data from the first round of competition assays (days $0-3$ in Figures $2 \mathbf{B}, \mathbf{C}$ ) because these measurements were unusually variable, and this variance was most likely an experimental artifact. Specifically, setting up the initial experiment took substantially longer than carrying out subsequent replatings as additional steps were required before seeding the cells. Since cells were kept for longer in suspension before the first round, they will have experienced more stress and potentially mortality. This means that results of the first round of competition assays are likely to be less reliable than results of subsequent rounds. For completeness, Supplementary Figures 5C,D show linear regression applied to the entire data set, including the first round.

\section{Carrying Capacities}

To find carrying capacities, we note that the phase 2 model can alternatively be parameterized as

$\frac{d L}{d t}=L r_{L, 2}\left(1-\frac{L+\beta W}{K_{L}}\right), \frac{d W}{d t}=W r_{W, 2}\left(1-\frac{\gamma L+W}{K_{W}}\right)$,

where the parameters are calculated as in Table 1. The carrying capacities $K_{W}$ and $K_{L}$ are the upper limits approached by the population sizes of $W$ and $L$, respectively, during phase 2 .

\section{Potential for Coexistence in vivo}

In a growing tumor, we expect cell-cell competition to be less than in our in vitro experiments, because, in the former, resources are continually replenished and waste materials removed by the host circulatory system. The evolutionary dynamics will then mostly depend on the difference in intrinsic growth rates and interactions mediated by diffusible factors. Furthermore, during tumor growth, the dynamics may be better described by a frequency- rather than a density-dependent model. We can then describe the evolutionary dynamics within the framework of evolutionary game theory using the payoff matrix

$$
\left(\begin{array}{cc}
\beta_{L}-\gamma & \alpha_{L}-\gamma \\
\beta_{W} & \alpha_{W}
\end{array}\right)
$$

where $\alpha_{L}, \alpha_{W}<0$ denote the harm inflicted by $W$ on $L$ and $W$, respectively; $\beta_{L}, \beta_{W}>0$ are the benefits bestowed by $L$ to $L$ and $W$, respectively; and $\gamma>0$ is the difference between the intrinsic exponential growth rates. The relative values of the entries in the payoff matrix determine which game (for example, prisoner's dilemma or hawk-dove) is equivalent to the evolutionary dynamics.

The parameter values inferred for phase 2 of the competition assays imply

$$
\beta_{W}>\beta_{L}-\gamma>\alpha_{W}>\alpha_{L}-\gamma
$$

in which case the evolutionary dynamics are equivalent to a prisoner's dilemma game for which $W$ is the only evolutionarily stable strategy (ESS). This means that $W$ (4T07) can invade and stably replace a population of $L$ (168).

If instead $\alpha_{L}-\gamma>\alpha_{W}$ then the payoff matrix defines a hawkdove game that permits coexistence. In this scenario, $W$ harms itself more than it harms $L$, and this difference outweighs $W^{\prime} s$ higher intrinsic growth rate. This could happen, for example, if harmful factors produced by $W$ imperfectly diffuse, so that $W$ cells experience a higher concentration than $L$ cells. At the mixed ESS, the $W$ proportion is

$$
\frac{\alpha_{W}-\alpha_{L}+\gamma}{\alpha_{W}-\alpha_{L}+\beta_{L}-\beta_{W}} .
$$

However, if additionally $\beta_{L}-\beta_{W}>\gamma$ (so that $L$ benefits itself more than it benefits $W$, and this difference outweighs $W^{\prime} s$ higher intrinsic growth rate) then coexistence again becomes impossible as the game again becomes a prisoner's dilemma but with $L$ as the ESS.

In a resource-poor environment, we might describe the evolutionary dynamics using the payoff matrix

$$
\left(\begin{array}{cc}
\beta_{L}-\gamma & \alpha_{L}-\gamma \\
\beta_{W}-\delta & \alpha_{W}-\delta
\end{array}\right)
$$

where $\delta$ is the reduction in $W^{\prime} s$ intrinsic growth rate due to the degraded environment (as inferred for phase 3 of our 96-h competition assays). This scenario favors $L$ and suggests that $L$ may be the ESS in a resource-poor environment, such as hypoxic regions within a tumor.

\section{DATA AVAILABILITY STATEMENT}

The raw data supporting the conclusions of this article will be made available by the authors, without undue reservation.

\section{AUTHOR CONTRIBUTIONS}

$\mathrm{UH}, \mathrm{MH}$, and PL conceived the study. VW, CR, and PL designed and performed the experiments and analyzed the data. RN designed and performed the mathematical modeling and mathematical analysis. $\mathrm{RN}, \mathrm{UH}, \mathrm{MH}$, and $\mathrm{PL}$ wrote the manuscript with contributions from VW and CR. All authors contributed to the article and approved the submitted version.

\section{FUNDING}

The authors wish to thank HTE "HetCoLi" (HTE20161) and ITMO "Physique Cancer" (CanEvolve PC201306) for funding. $\mathrm{RN}$ acknowledges support from the National Cancer Institute of the National Institutes of Health under Award Number U54CA217376. The content is solely the responsibility of the authors and does not necessarily represent the official views of the National Institutes of Health. CR was supported by the French Infrastructure for Integrated Structural Biology (FRISBI) (grant no. ANR-10-INSB-05). MH thanks the McDonnell Foundation for funding (Studying Complex Systems research award 220020294). 


\section{ACKNOWLEDGMENTS}

We are grateful to Emie Quissac and Yasser Kerboua for their excellent technical assistance, and to Artem Kaznatcheev for helpful conversations.

\section{SUPPLEMENTARY MATERIAL}

The Supplementary Material for this article can be found online at: https://www.frontiersin.org/articles/10.3389/fevo.2021. 675638/full\#supplementary-material

Supplementary Figure 1 | (A,B) Growth dynamics of subclones under homotypic and heterotypic conditions. $10^{5}$ Cells were seeded at a 3:1 (A) or 1:4 (B) ratios in homotypic (parental and GFP expressing derivative of the same cell line) or heterotypic (different cell lines, one expressing GFP) co-cultures and harvested and replated at the initial densities ( $10^{5}$ cells/plate) at indicated times. The ratios of GFP-labeled to unlabeled cells were estimated by flow cytometry. The results represent data from three independent experiments and are shown as mean \pm SEM.

Supplementary Figure 2 | (A) Apoptosis quantification of subclones under homotypic and heterotypic conditions. A total of $10^{5}$ cells were seeded. 168G cells were co-cultured with either the 168P (homotypic) or 4T07P (heterotypic) cells at a 1:1 ratio for 4 days and harvested. Apoptosis was quantified by flow cytometry following Annexin- $\mathrm{V}$ staining. ns: not significant. (B) S phase quantification of subclones under homotypic and heterotypic conditions. A total of $10^{5}$ cells were seeded. $168 \mathrm{G}$ cells were co-cultured with either the $168 \mathrm{P}$ (homotypic) or 4T07P (heterotypic) cells at a 1:1 ratio for 4 days. Before harvesting at day 4 cells were labeled by a $2 \mathrm{~h}$ pulse of EdU and the fraction of cells in the $\mathrm{S}$ phase was determined by flow cytometry. ${ }^{*} p<0.05,{ }^{* *} p<0.01$.

Supplementary Figure 3 | Growth dynamics of subclones at low and high density. Experiments were performed as in Figure 3B. Cells were grown in heterotypic conditions at a starting ratio of 1:1. Cells were seeded either at low density (50k) or high density (150k), diluted, and quantified every 3 days. At low density, cells do not reach confluence before replating. The results represent data from three independent experiments and are shown as mean \pm SEM.

\section{REFERENCES}

Amirouchene-Angelozzi, N., Swanton, C., and Bardelli, A. (2017). Tumor evolution as a therapeutic target. Cancer Discov. 7, 805-817. doi: 10.1158/2159. 8290.cd-17-0343

Archetti, M., Ferraro, D. A., and Christofori, G. (2015). Heterogeneity for IGF-II production maintained by public goods dynamics in neuroendocrine pancreatic cancer. Proc. Natl. Acad. Sci. USA 112, 1833-1838. doi: 10.1073/ pnas. 1414653112

Aslakson, C. J., Rak, J. W., Miller, B. E., and Miller, F. R. (1991). Differential influence of organ site on three subpopulations of a single mouse mammary tumor at two distinct steps in metastasis. Internat. J. Cancer 47, 466-472. doi: 10.1002/ijc.2910470327

Axelrod, R., Axelrod, D. E., and Pienta, K. J. (2006). Evolution of cooperation among tumor cells. Proc. Natl. Acad. Sci. USA 103, 13474-13479. doi: 10.1073/ pnas.0606053103

Beckman, R. A., Kareva, I., and Adler, F. R. (2020). How Should Cancer Models Be Constructed? Cancer Control 27, 1-12.

Calbo, J., van Montfort, E., Proost, N., van Drunen, E., Beverloo, H. B., Meuwissen, R., et al. (2011). A functional role for tumor cell heterogeneity in a mouse model of small cell lung cancer. Cancer Cell 19, 244-256. doi: 10.1016/j.ccr.2010. 12.021

Chapman, A., del Ama, L. F., Ferguson, J., Kamarashev, J., Wellbrock, C., and Hurlstone, A. (2014). Heterogeneous tumor subpopulations cooperate to drive invasion. Cell Rep. 8, 688-695. doi: 10.1016/j.celrep.2014.06.045
Supplementary Figure 4 | (A) Expression levels of the slc16A family transporter genes in 168FARN. RT-QPCR analysis was performed on 168FARN RNA for Mct2, Mct1, Mct3, and Mct4 genes and normalized to HPRT. Relative expression levels were compared to Mct2. (B) Slc16A1 expression in both subclones. Slc16A1 RNA levels were monitored by RT-QPCR, normalized with HPRT and adjusted relative to levels in 168FARN cells. (C) Influence of Slc16A1 expression by $\beta$-hydroxybutyrate. Experiment was performed as in Figure 5B. Slc16A1 RNA levels were quantified as in panel $(\mathbf{A})$ and adjusted relative to levels in control condition. ${ }^{* * *} p<0.001$.

Supplementary Figure 5 | (A) Mean net growth rate difference (gain function) versus initial 4T07 proportion in phases 1 and 2 (purple) and phases 1, 2, and 3 (green). Each point corresponds to the outcome of a competition assay. Regression lines are shown with 95\% confidence intervals. (B) Mean net growth rate difference versus initial 4T07 proportion in phase 2 (purple) and phases 2 and 3 (green). This data set was obtained from the data shown in panel (A) by adjusting for exponential growth in phase 1 (see section "Materials and Methods"). (C) The same as panel (A) but including results for the first round of competition assays (days 0-3). First-round measurements were excluded from analyses as they were unusually variable and unreliable due to an experimental artifact (see section "Materials and Methods"). (D) The same as panel (B) but including results for the first round of competition assays (days 0-3).

Supplementary Figure 6 | Relationship between population dynamics and net growth rates. The net growth rate of each cell type (right column) is the derivative of its log-transformed growth curve (left column). (A) Mathematical model dynamics. From the dynamical model, net growth rates can be found precisely by evaluating differential equation terms. The model was parameterized with values inferred from data (Table 1) and initiated with a 3:1 ratio of 168-4T07. (B) Empirical dynamics. From time-lapse data, net growth rates can be approximated as local gradients (difference quotients). In this example, we estimated net growth rates from smoothed growth curves by calculating difference quotients across a 5-h span. Smoothed growth curves (not shown) were obtained by computing running medians with a 5-h span. Since we did not use heterotypic time-lapse data for parameter inference, the resemblance between the two rows of this figure contributes to validating our model. The data in panel $\mathbf{( B )}$ is the same as in

Figures 3A,B

Supplementary Figure 7 | Mathematical relationships relevant to our methods. The diagram illustrates several equivalent ways of calculating the mean growth rate difference (gain function, blue) from the parameterized dynamical model (red). Also shown is our method of calculating the gain function from competition assay data (orange).

Cheng, C.-W., Biton, M., Haber, A. L., Gunduz, N., Eng, G., Gaynor, L. T., et al. (2019). Ketone Body Signaling Mediates Intestinal Stem Cell Homeostasis and Adaptation to Diet. Cell 178, 1115.e-1131.e.

Cleary, A. S., Leonard, T. L., Gestl, S. A., and Gunther, E. J. (2014). Tumour cell heterogeneity maintained by cooperating subclones in Wnt-driven mammary cancers. Nature 508, 113-117. doi: 10.1038/nature13187

De Bock, K., Cauwenberghs, S., and Carmeliet, P. (2011). Vessel abnormalization: another hallmark of cancer?: Molecular mechanisms and therapeutic implications. Curr. Opinion Genet. Dev. 21, 73-79. doi: 10.1016/j.gde.2010. 10.008

De Palma, M., Biziato, D., and Petrova, T. V. (2017). Microenvironmental regulation of tumour angiogenesis. Nat. Rev. Cancer. 17, 457-474. doi: 10.1038/ nrc.2017.51

Dexter, D. L., Kowalski, H. M., Blazar, B. A., Fligiel, Z., Vogel, R., and Heppner, G. H. (1978). Heterogeneity of tumor cells from a single mouse mammary tumor. Cancer Res. 38, 3174-3181.

Farrokhian, N., Maltas, J., Ellsworth, P., Durmaz, A., Dinh, M., Hitomi, M., et al. (2020). Dose dependent evolutionary game dynamics modulate competitive release in cancer therapy. bioRxiv 2020:18.303966.

Gatenby, R. A., and Brown, J. (2017). Mutations, evolution and the central role of a self-defined fitness function in the initiation and progression of cancer. Biochim. Biophy.Acta 1867, 162-166. doi: 10.1016/j.bbcan.2017.03.005

Gatenby, R. A., and Brown, J. (2020). Integrating evolutionary dynamics into cancer therapy. Nat. Rev. Clin. Oncol. 17, 675-686. doi: 10.1038/s41571-0200411-1 
Grabacka, M. M., Wilk, A., Antonczyk, A., Banks, P., Walczyk-Tytko, E., Dean, M., et al. (2016). Fenofibrate Induces Ketone Body Production in Melanoma and Glioblastoma Cells. Front. Endocrinol. 2016:7. doi: 10.3389/fendo.2016.00005

Greaves, M., and Maley, C. C. (2012). Clonal evolution in cancer. Nature 481, 306-313.

Grivennikov, S. I. (2013). IL-11: a prominent pro-tumorigenic member of the IL-6 family. Cancer Cell 24, 145-147. doi: 10.1016/j.ccr.2013.07.018

Henke, J., Willker, W., Engelmann, J., and Leibfritz, D. (1996). Combined extraction techniques of tumour cells and lipid/phospholipid assignment by two dimensional NMR spectroscopy. Anticancer Res. 16, 1417-1427.

Heppner, G. H. (1984). Tumor heterogeneity. Cancer Res. 44, 2259-2265.

Huang, C.-K., Chang, P.-H., Kuo, W.-H., Chen, C.-L., Jeng, Y.-M., Chang, K.-J., et al. (2017). Adipocytes promote malignant growth of breast tumours with monocarboxylate transporter 2 expression via $\beta$-hydroxybutyrate. Nat. Comm. 8:14706.

Janiszewska, M., Tabassum, D. P., Castaño, Z., Cristea, S., Yamamoto, K. N., Kingston, N. L., et al. (2019). Subclonal cooperation drives metastasis by modulating local and systemic immune microenvironments. Nat. Cell Biol. 21, 879-888. doi: 10.1038/s41556-019-0346-x

Kaznatcheev, A. (2017). Two conceptions of evolutionary games: reductive vs effective. bioRxiv 2017:231993.

Kaznatcheev, A., Peacock, J., Basanta, D., Marusyk, A., and Scott, J. G. (2019). Fibroblasts and alectinib switch the evolutionary games played by non-small cell lung cancer. Nat. Ecol. Evol. 3, 450-456. doi: 10.1038/s41559-018-0768-z

Lu, P., Weaver, V. M., and Werb, Z. (2012). The extracellular matrix: A dynamic niche in cancer progression. J. Cell Biol. 196, 395-406. doi: 10.1083/jcb. 201102147

Maley, C. C., Aktipis, A., Graham, T. A., Sottoriva, A., Boddy, A. M., Janiszewska, M., et al. (2017). Classifying the evolutionary and ecological features of neoplasms. Nat. Rev. Cancer 17, 605-619. doi: 10.1038/nrc.2017.69

Marusyk, A., Tabassum, D. P., Altrock, P. M., Almendro, V., Michor, F., and Polyak, K. (2014). Non-cell-autonomous driving of tumour growth supports sub-clonal heterogeneity. Nature 514, 54-58. doi: 10.1038/nature13556

Merlo, L. M. F., Pepper, J. W., Reid, B. J., and Maley, C. C. (2006). Cancer as an evolutionary and ecological process. Nat. Rev. Cancer 6, 924-935. doi: $10.1038 /$ nrc2013

Miller, B. E., Miller, F. R., Wilburn, D., and Heppner, G. H. (1988). Dominance of a tumor subpopulation line in mixed heterogeneous mouse mammary tumors. Cancer Res. 48, 5747-5753.

Naffar-Abu Amara, S., Kuiken, H. J., Selfors, L. M., Butler, T., Leung, M. L., Leung, C. T., et al. (2020). Transient commensal clonal interactions can drive tumor metastasis. Nat. Comm. 11:1.

Newman, J. C., and Verdin, E. (2017). $\beta$-Hydroxybutyrate: A Signaling Metabolite. Ann. Rev. Nutri. 37, 51-76. doi: 10.1146/annurev-nutr-071816-064916

Noble, R., Burley, J. T., Le Sueur, C., and Hochberg, M. E. (2020). When, why and how tumour clonal diversity predicts survival. Evol. Appl. 13, 1558-1568. doi: 10.1111/eva.13057

Nowell, P. (1976). The clonal evolution of tumor cell populations. Science 194, 23-28. doi: 10.1126/science.959840

Ohsawa, S., Sato, Y., Enomoto, M., Nakamura, M., Betsumiya, A., and Igaki, T. (2012). Mitochondrial defect drives non-autonomous tumour progression through Hippo signalling in Drosophila. Nature 490, 547-551. doi: 10.1038/ nature 11452

Poff, A. M., Ari, C., Arnold, P., Seyfried, T. N., and D'Agostino, D. P. (2014). Ketone supplementation decreases tumor cell viability and prolongs survival of mice with metastatic cancer. Internat. J. Cancer 135, 1711-1720. doi: 10.1002/ijc. 28809

Poff, A. M., Ari, C., Seyfried, T. N., and D'Agostino, D. P. (2013). The ketogenic diet and hyperbaric oxygen therapy prolong survival in mice with systemic metastatic cancer. PLoS One 8:e65522. doi: 10.1371/journal.pone.006 5522

Quail, D. F., and Joyce, J. A. (2013). Microenvironmental regulation of tumor progression and metastasis. Nat. Med. 19, 1423-1437. doi: 10.1038/nm.3394

Robinson, S. P., and Jordan, V. C. (1989). The paracrine stimulation of MCF-7 cells by MDA-MB-231 cells: possible role in antiestrogen failure. Eur. J. Cancer Clin. Oncol. 25, 493-497. doi: 10.1016/0277-5379(89)90262-9

Shakery, A., Pourvali, K., Ghorbani, A., Sadat, F. S., and Zand, H. (2018). Beta-Hydroxybutyrate Promotes Proliferation, Migration and Stemness in a Subpopulation of 5FU Treated SW480 Cells: Evidence for Metabolic Plasticity in Colon Cancer. Asian Pacific J. Cancer Prevent. 19, 3287-3294. doi: 10.31557/ apjcp.2018.19.11.3287

Shimazu, T., Hirschey, M. D., Newman, J., He, W., Shirakawa, K., Le Moan, N., et al. (2013). Suppression of oxidative stress by $\beta$-hydroxybutyrate, an endogenous histone deacetylase inhibitor. Science 339, 211-214. doi: 10.1126/ science. 1227166

Soetaert, K., Petzoldt, T., and Setzer, R. W. (2010). Solving Differential Equations in R: Package deSolve. J. Statist. Software 33, 1-25. doi: 10.1137/1.97808987196 35.ch1

Tabassum, D. P., and Polyak, K. (2015). Tumorigenesis: it takes a village. Nat. Rev. Cancer 15, 473-483. doi: 10.1038/nrc3971

Viossat, Y., and Noble, R. (2021). A theoretical analysis of tumour containment. Nat. Ecol. Evol. 5, 826-835. doi: 10.1038/s41559-021-01428-w

Vishwakarma, M., and Piddini, E. (2020). Outcompeting cancer. Nat. Rev. Cancer 20, 187-198. doi: 10.1038/s41568-019-0231-8

Waclaw, B., Bozic, I., Pittman, M. E., Hruban, R. H., Vogelstein, B., and Nowak, M. A. (2015). A spatial model predicts that dispersal and cell turnover limit intratumour heterogeneity. Nature 525, 261-264. doi: 10.1038/nature14971

Wang, Q., Zhou, Y., Rychahou, P., Fan, T. W.-M., Lane, A. N., Weiss, H. L., et al. (2017). Ketogenesis contributes to intestinal cell differentiation. Cell Death Diff. 24, 458-468. doi: $10.1038 / \mathrm{cdd} .2016 .142$

Yang, J., and Moses, M. A. (2009). Lipocalin 2: A multifaceted modulator of human cancer. Cell Cycle 8, 2347-2352. doi: 10.4161/cc.8.15.9224

Conflict of Interest: The authors declare that the research was conducted in the absence of any commercial or financial relationships that could be construed as a potential conflict of interest.

Publisher's Note: All claims expressed in this article are solely those of the authors and do not necessarily represent those of their affiliated organizations, or those of the publisher, the editors and the reviewers. Any product that may be evaluated in this article, or claim that may be made by its manufacturer, is not guaranteed or endorsed by the publisher.

Copyright (c) 2021 Noble, Walther, Roumestand, Hochberg, Hibner and Lassus. This is an open-access article distributed under the terms of the Creative Commons Attribution License (CC BY). The use, distribution or reproduction in other forums is permitted, provided the original author(s) and the copyright owner(s) are credited and that the original publication in this journal is cited, in accordance with accepted academic practice. No use, distribution or reproduction is permitted which does not comply with these terms. 